\title{
Estrategias de base agroecológica para y con agricultores familiares de la región Pampeana. Construyendo faros agroecológicos
}

Ambiente y extensión universitaria /

Intervenciones

\section{Ramón Cieza}

cieza@agro.unlp.edu.ar

(iD) orcid.org/0000-0001-8645-3155

Griselda Sánchez Vallduví

sanchezandreoli@gmail.com

(iD) orcid.org/0000-0003-3668-1656

Nora Tamagno

Itamagno@agro.unlp.edu.ar

(iD) orcid.org/0000-0002-4346-875X

RECEPCIÓN: 13/04/21

ACEPTACIÓN FINAL: 03/06/21

\section{Resumen}

Este trabajo describe y analiza un proceso de intervención realizada por un equipo interdisciplinario de la Facultad de Ciencias Agrarias y Forestales de la Universidad Nacional de la Plata, Argentina, cuyo objetivo fue difundir, desarrollar y evaluar estrategias y tecnologías de base agroecológica apropiadas para y con productores familiares de la región Pampeana. Se describen los aportes que realizó el proyecto a partir del acompañamiento, intercambio de saberes y fortalecimiento de sistemas productivos agroecológicos en el territorio. Respecto del desarrollo de la experiencia, se analiza la importancia de generar metodologías apropiadas para ampliar los conocimientos acerca del funcionamiento y puesta en práctica de sistemas de base agroecológica. Uno de los aprendizajes más significativos a partir de la experiencia fue la necesidad de generar cambios profundos en las estrategias de investigación, extensión y formación de profesionales con una nueva mirada, en sintonía con la incorporación del enfoque agroecológico como nuevo paradigma de las ciencias agropecuarias.

Palabras clave: faros agroecológicos; extensión universitaria; diálogo de saberes; formación agroecológica.

\section{Santiago Sarandón}

Comisión de Investigaciones Científicas

de la Provincia de Buenos Aires, Argentina. sarandon@agro.unlp.edu.ar

(iD) orcid.org/0000-0001-5197-3652

Universidad Nacional de La Plata, Argentina.

\section{Estratégias de base agroecológica para e com agricultores familiares na região Pampeana. Construindo faróis agroecológicos}

\section{Resumo}

This work describes and analyzes an intervention process carried out by an interdisciplinary team of the Faculty of Agricultural and Forest Sciences of the National University of La Plata, Argentina, whose objective was to disseminate, develop and evaluate appropriate agroecological strategies and technologies for and with family producers from the Pampean region. The contributions made by the project are described from the accompaniment, exchange of knowledge and strengthening of agroecological productive systems in the territory. Based on the development of experience, the importance of generating appropriate methodologies to expand knowledge on the operation and implementation of agroecological systems is analyzed. One of the most significant lessons learned from the experience was the need to generate profound changes in the strategies of research, extension, and training of professionals with a new perspective, in tune with the incorporation of the agroecological approach as a new paradigm of science agricultural.

Keywords: agroecological lighthouses; college extension; dialogue of knowledge; agroecological training.
Este trabalho descreve e analisa um processo de intervenção realizado por uma equipe interdisciplinar da Faculdade de Ciências Agrárias e Florestais da Universidade Nacional de La Plata, Argentina, cujo objetivo foi divulgar, desenvolver e avaliar estratégias e tecnologias agroecológicas adequadas para e com produtores familiares da região Pampeana. As contribuições do projeto são descritas a partir do acompanhamento, troca de conhecimentos e fortalecimento dos sistemas produtivos agroecológicos no território. Com base no desenvolvimento da experiência, analisa-se a importância da geração de metodologias adequadas para ampliar o conhecimento sobre a operação e implantação de sistemas agroecológicos. Uma das aprendizagens mais significativa adquirida com a experiência foi a necessidade de gerar mudanças profundas nas estratégias de pesquisa, extensão e formação de profissionais com uma nova perspectiva, em sintonia com a incorporação da abordagem agroecológica como um novo paradigma da ciência agrícola.

Palavras-chave: faróis agroecológicos; extensão da faculdade; diálogo de saberes; treinamento agroecológico.

Para citación de este artículo: Cieza, R.; Sánchez Vallduví, G.; Tamagno, N. y Sarandón, S. (2021). Estrategias de base agroecológica para y con agricultores familiares de la Región Pampeana. Construyendo faros agroecológicos. +E: Revista de Extensión Universitaria, 11(14), e0005. doi: 10.14409/extension.2021.14.Ene-Jun.e0005 


\section{Introducción}

Desde hace más de treinta años se ha venido consolidando en Argentina, y en la región Pampeana como epicentro, un modelo de agricultura industrial hegemónica que busca aumentos de productividad mediante el uso de genotipos mejorados de cultivos y animales, con un alto uso de insumos químicos (pesticidas y fertilizantes) y energía (principalmente fósil). A pesar de los altos rendimientos logrados y su "aparente" rentabilidad, este modelo está en crisis debido a su "insustentabilidad" y su inaplicabilidad a un amplio número de agricultores (Sarandón y Flores, 2014). La búsqueda de una agricultura sustentable que disminuya el uso de insumos (caros y riesgosos) requiere un nuevo paradigma de las ciencias agropecuarias. La agroecología propone una novedosa mirada sobre la actividad agropecuaria y toma como objetivos la producción y distribución de alimentos sanos y diversos, la equidad social y la sustentabilidad ecosistémica de las condiciones de reproducción de la vida a nivel local, regional y global (SAAE, 2020). Si bien la agroecología cuenta con un gran potencial para abordar los múltiples desafíos que enfrenta la agricultura actual, no es un modelo ampliamente difundido en los agricultores. Existen condicionantes estructurales para su expansión pero también otros aspectos vinculados a la forma de generación, rescate e intercambio de conocimientos entre productores, técnicos e instituciones.

Este trabajo describe y analiza el proyecto "Desarrollo y difusión de estrategias de base agroecológica para y con productores familiares de la región Pampeana", el cual busca aportar soluciones a la problemática antes planteada. El mismo fue realizado en el marco de los Proyectos Institucionales para el Desarrollo Tecnológico y Social (PITS) de la Facultad de Ciencias Agrarias y Forestales de la Universidad Nacional de La Plata (UNLP), Argentina, entre los años 2017 y 2019. Los objetivos del proyecto apuntaron a desarrollar, evaluar y difundir estrategias y tecnologías de bajo uso de insumos, de base agroecológica, apropiadas para sistemas de producción familiar de la región Pampeana.

La estructura del trabajo contempla una primera parte donde se describen las acciones llevadas a cabo en el proceso de intervención. Luego, se analizan los avances obtenidos en el proyecto en cuanto a la generación de conocimiento con los productores, el acompañamiento y consolidación de "faros agroecológicos y la formación de profesionales con una perspectiva agroecológica. De manera simultánea, se reflexiona sobre el proceso, se plantean aciertos, dificultades y desafíos, y en las conclusiones se analizan los principales aprendizajes del proyecto así como aspectos fundamentales para continuar trabajando a futuro.

\section{Acciones realizadas en el marco del proyecto}

El proyecto desarrollado entre los años 2017 y 2019 integró diferentes grupos de trabajo de la Facultad que venían llevando a cabo actividades de investigación y/o extensión en temáticas vinculadas a la agroecología en sistemas productivos, instituciones y organizaciones. Estos grupos estaban conformados por docentes de diversas asignaturas de la Facultad de Ciencias Agrarias y Forestales, tales como Agroecología, Oleaginosas y Cultivos Regionales, Introducción a las Ciencias Agrarias, Producción Animal II, Agroindustrias, Fruticultura y Socioeconomía; docentes de la Facultad de Ciencias Veterinarias; graduados; estudiantes y representantes de diferentes instituciones y organizaciones locales. Se apuntó a generar líneas de trabajo y proyectos de investigación de manera conjunta con los agricultores fami- 
liares de la región con el objetivo de recoger sus inquietudes y demandas. Por otra parte, se propendió a lograr un mayor involucramiento por parte de la Facultad en las problemáticas de la producción familiar, consolidando vínculos existentes y promoviendo nuevos lazos entre los diferentes actores participantes.

Las experiencias se desarrollaron en distintos territorios, cada uno de ellos con particularidades socioproductivas: a) Áreas periurbanas con sistemas hortícolas al aire libre -productores de los partidos de La Plata, Berisso, Cañuelas y San Vicente; b) Producción lechera familiar en los partidos de San Vicente, Cañuelas y Magdalena; y c) Producciones extensivas de tipo mixto de partidos del interior de la provincia de Buenos Aires. Se trabajó con productores y productoras heterogéneos, siendo el patrón común su pertenencia a la agricultura familiar. Algunos de ellos formaban parte de organizaciones de productores que fomentan la agroecología como alternativa al modelo agroindustrial ${ }^{1}$ y en otros casos no contaban con un grupo de pertenencia, por lo que los motivaba una cuestión personal en la transformación del modelo productivo.

Las tareas se iniciaron con la realización de un diagnóstico participativo para conocer la situación actual, identificar causas y recursos disponibles con la intención de buscar alternativas que aporten al proceso de conversión. Para ello se hizo un encuentro inicial del cual participaron productores de San Vicente, Cañuelas, Berisso y La Plata, junto a docentes-investigadores-extensionistas. En el mismo se rescataron inquietudes y problemáticas que se asociaron a las dificultades para llevar a cabo los procesos de cambio en el manejo del suelo, control de malezas y plagas, comercialización de la producción, entre otras. En el taller surgió también la necesidad de involucrar a otros grupos con problemáticas comunes y articular actividades con la Universidad para trabajarlas.

En el territorio cercano a la Universidad (La Plata, Berisso, Magdalena, San Vicente y Cañuelas) se realizaron tres charlas de difusión, dos talleres y dos jornadas de trabajo a campo con una asistencia mínima de 25 y máxima de 70 participantes entre productores, técnicos, docentes y estudiantes. Los talleres se desarrollaron en la Facultad de Ciencias Agrarias y las jornadas en campos de productores en proceso de conversión a la agroecología. En estas actividades se trabajó en las potencialidades y limitantes para el desarrollo de sistemas productivos agroecológicos. Como complemento, se trabajó en la consolidación de experiencias agroecológicas locales que puedan ser demostrativas para otros productores (faros agroecológicos) a partir del apoyo técnico, el relevamiento de datos y la sistematización de la experiencia.

Por otra parte, en el interior de la provincia de Buenos Aires se llevaron a cabo tres talleres en los municipios de Guaminí y Lincoln en el marco del vínculo del proyecto con la Red Nacional de Municipios y Comunidades que fomentan la Agroecología (RENAMA). De los talleres participaron entre 60 y 90 personas distribuidas en grupos constituidos por productores y productoras, investigadores e investigadoras, extensionistas, becarios y becarias, y público en general. Estos talleres apuntaron a detectar los principales "cuellos de botella" o impedimentos para avanzar en los procesos de conversión hacia sistemas de base agroecológica. 


\section{Resultados obtenidos y una reflexión del proceso}

El primer logro obtenido en el marco del proyecto fue la posibilidad de integrar grupos que venían trabajando atomizados, con escasa articulación entre sí. El primer desafío fue, entonces, complementar las líneas de trabajo en desarrollo con la incorporación de nuevos aspectos y la sinergia por el intercambio entre los grupos, con experiencias, visiones y conocimientos variados. El taller inicial, que contó con la totalidad de los integrantes del proyecto, tuvo como objetivo hacer un diagnóstico participativo y definir las actividades que debía contener el proyecto, con lo cual se pudo delinear, detectar y/o visibilizar una serie de propuestas de trabajo. ${ }^{2} \mathrm{~A}$ grandes rasgos, las mismas apuntaron a: a) la difusión de la propuesta agroecológica; b) la generación e intercambio de conocimiento sobre la implementación de la agroecología en los diferentes tipos de producciones; c) el fomento de sistemas productivos agroecológicos en el territorio.

En cuanto a la difusión de la agroecología entre productores, técnicos, estudiantes y público en general, se trabajó por medio de charlas y talleres en la Facultad, jornadas en las localidades y en campo de productores. Esto permitió la sensibilización sobre los problemas causados por el modelo productivo de agricultura industrial y acerca de la necesidad y posibilidad de producir de manera alternativa a través del enfoque agroecológico. Las charlas tuvieron un rol clave en la generación de nuevas experiencias y en el creciente interés de diversos actores relacionados con el medio productivo, entes gubernamentales, ${ }^{3}$ estudiantes y profesionales de diversas áreas, por vincularse y formarse para fomentar y promover una producción agroecológica.

\section{La generación de conocimiento con los productores}

De las demandas del taller inicial, uno de los ejes a trabajar fue la necesidad de generar conocimiento de validez local que aportara a los procesos de conversión hacia sistemas de base agroecológica que se proponían en los territorios. Tal como plantean Altieri y Nichols (2019), un desafío es la traducción de los principios agroecológicos en estrategias prácticas para la gestión del suelo, el agua y la biodiversidad, para mejorar la producción y la resiliencia de los sistemas productivos. La articulación entre la investigación y la extensión fue una de las líneas de trabajo. Para ello se efectuaron talleres con productores, estudiantes y docentes-investigadores-extensionistas a los fines de detectar y priorizar líneas y metodologías de investigación para afrontar las problemáticas que surgían de los procesos de conversión hacia sistemas de base agroecológica. Los talleres permitieron identificar una diversidad de temáticas, muchas de ellas expresadas en forma amplia. Uno de los aspectos más relevantes de los mismos fue la manifestación de muchos productores y productoras acerca de lo importante y novedoso que resultaba para ellos que se les preguntara cuáles eran sus problemas o dudas. Que la Universidad estuviese presente preguntando y no enseñando fue

2) Los estudiantes tuvieron una activa participación en la preparación previa, el registro de datos y en la coordinación de los grupos.

3) Tal como plantean Patrouilleau et al. (2017), no existe en Argentina una política pública integral a favor de la agroecología pero sí programas e instrumentos que favorecieron su desarrollo, como el Programa Prohuerta, líneas de investigación y extensión dentro de INTA y las universidades. Recientemente, es destacable mencionar la creación de la Dirección Nacional de Agroecología dentro del Ministerio de Agricultura de la Nación, juntamente con la incorporación del enfoque en el Ministerio de Desarrollo Agrario de la Provincia de Buenos Aires. 
toda una novedad. Seguramente esa necesidad existió siempre, pero no podía ser expresada porque no existía ese encuentro (con esa actitud).

En los talleres se detectaron necesidad de saberes para desarrollar los cambios hacia sistemas de base agroecológica. A esos saberes los hemos clasificado en tres categorías: los que ostentan carácter más general, los que se asocian al funcionamiento de procesos en los agroecosistemas, y los que se vinculan con el desarrollo de prácticas (Tabla 1).

Tabla 1. Saberes necesarios para la conversión a sistemas de base agroecológica sistematizados a partir del trabajo en taller

\begin{tabular}{|c|c|c|}
\hline Generales & Asociados a procesos & Asociados a prácticas \\
\hline $\begin{array}{l}\text { Demostrar los perjuicios que ocasiona } \\
\text { el modelo convencional. }\end{array}$ & $\begin{array}{l}\text { Estudiar la dinámica del desarrollo de las } \\
\text { comunidades de malezas y estrategias para } \\
\text { el manejo ecológico de las mismas. }\end{array}$ & $\begin{array}{l}\text { Conocer sobre asociación de cultivos } \\
\text { de verano. }\end{array}$ \\
\hline $\begin{array}{l}\text { Discutir, validar y/o refutar tecnologías } \\
\text { foráneas. }\end{array}$ & $\begin{array}{l}\text { Conocer microorganismos y su, funciona- } \\
\text { miento como estrategias alternativas de } \\
\text { fertilización química. }\end{array}$ & $\begin{array}{l}\text { Cómo hacer asociaciones, por ejemplo, } \\
\text { trébol con trigo. }\end{array}$ \\
\hline $\begin{array}{l}\text { Rescatar prácticas antiguas y/o alternati- } \\
\text { vas a lo convencional. }\end{array}$ & $\begin{array}{l}\text { Conocer cuáles son los manejos adecuados } \\
\text { de biodiversidad y cómo manejarlos. }\end{array}$ & $\begin{array}{l}\text { Conocer sobre plantas autóctonas, } \\
\text { usos, opciones de cultivo. }\end{array}$ \\
\hline $\begin{array}{l}\text { Realizar otras formas de análisis } \\
\text { económico que contemple los "beneficios } \\
\text { ambientales". }\end{array}$ & $\begin{array}{l}\text { Entender cómo interactúan las plantas. } \\
\text { ¿Qué aporta cada planta al sistema? }\end{array}$ & $\begin{array}{l}\text { Estudio de malezas y su uso como } \\
\text { indicadoras de calidad de sitio. }\end{array}$ \\
\hline $\begin{array}{l}\text { Asociar las investigaciones a mercados } \\
\text { de cercanía. }\end{array}$ & Estudiar el reciclaje de nutrientes. & $\begin{array}{l}\text { Manejo de pastizales naturales, com- } \\
\text { posición. }\end{array}$ \\
\hline Rescatar especies que hoy no se utilizan. & $\begin{array}{l}\text { Entender cómo trabajan el suelo } \\
\text { y los microorganismos. }\end{array}$ & $\begin{array}{l}\text { Cómo multiplicar semillas (variedades } \\
\text { aptas para la producción. }\end{array}$ \\
\hline $\begin{array}{l}\text { Desarrollar estrategias para revertir el } \\
\text { daño ecológico. }\end{array}$ & & $\begin{array}{l}\text { ¿Cómo evaluar integralmente la calidad } \\
\text { del suelo (biológico)? }\end{array}$ \\
\hline Cómo hacer planteos solo agrícolas. & $\begin{array}{l}\text { Conocer cuál es el rol y uso del árbol } \\
\text { en los sistemas pampeanos. }\end{array}$ & $\begin{array}{l}\text { Desarrollar investigaciones en sistemas } \\
\text { silvopastoriles. }\end{array}$ \\
\hline \multirow[t]{2}{*}{$\begin{array}{l}\text { Investigar sobre certificación participativa. } \\
\text { Certificación bromatológica. }\end{array}$} & & $\begin{array}{l}\text { Generar tecnologías de manejo del agua } \\
\text { para la agricultura familiar. }\end{array}$ \\
\hline & $\begin{array}{l}\text { Conocer cómo funcionan los corredores } \\
\text { biológicos y sus utilidades en el sistema. }\end{array}$ & $\begin{array}{l}\text { Investigación en variedades nativas, } \\
\text { semillas, comportamientos, consociacio- } \\
\text { nes, cualidades. }\end{array}$ \\
\hline
\end{tabular}

Fuente: elaboración propia sobre la base de talleres realizados en Guaminí (2017), La Plata y Lincoln (2018).

A pesar del valor que tiene haber logrado un listado de dudas y preguntas, su transformación en temas de investigación necesita de una mayor elaboración, capacidad y metodologías que permitan clarificar cómo realizar el proceso investigativo. Esto requiere un proceso más largo en el que se consensuen los temas a abordar con compromisos asumidos por productores y productoras, investigadores e investigadoras, de la tarea a llevar a cabo. Además, es preciso priorizar las temáticas, dado que un listado amplio con diferentes grados de complejidad y niveles de abordaje dificulta operativizar líneas de investigación. ${ }^{4}$ Por otra parte, el estudio de estas problemáticas, no lineales, requiere ampliar y consolidar equipos 
interdisciplinarios de investigación con enfoque agroecológico y adaptar los mecanismos actuales de evaluación de los científicos, excesivamente basados en el paper, que no facilita el desarrollo de áreas emergentes y complejas como la agroecología. A esto debe sumarse que se deben superar los prejuicios que existen sobre la agroecología como disciplina científica en gran parte de los investigadores de las ciencias agrarias.

La posibilidad de avanzar en un modelo agroecológico va acompañada del desarrollo de una agricultura familiar, la cual favorece procesos de construcción colectiva, que valoran los saberes tradicionales y promueven la conservación de los recursos naturales (Caporal y Costabeber, 2004; Caporal, 2009). En este sentido, comienzan a reconocerse, desde las universidades e instituciones de investigación y extensión agropecuaria, la importancia y el aporte que pueden hacer los sistemas de agricultura familiar, durante mucho tiempo invisibilizados o considerados ineficientes y abandonados, al proceso de rediseño de los agroecosistemas hacia sistemas sustentables. Sin embargo, su estudio no es sencillo ya que la complejidad propia de estos sistemas dificulta el abordaje desde instituciones formadas en el paradigma de la simplicidad y lo disciplinar. La experiencia de este proyecto permitió confirmar que en la articulación del conocimiento entre agricultores y agricultoras, organizaciones que trabajan en el territorio y el saber académico, la investigación desde un enfoque participativo y la extensión como proceso transformador a partir de la praxis cumplen una función fundamental (Cano et al., 2010). Por otra parte, el trabajo en el territorio con sistemas heterogéneos requiere consensuar miradas entre los actores, ya que no existe una experiencia única probada. También reafirma la importancia del trabajo conjunto de los profesionales de la extensión, quienes deben tener un compromiso y accionar que faciliten y estimulen la participación de los agricultores y agricultoras y contribuyan a mejorar el vínculo entre los actores que intervienen en el proceso de transformación colectiva. Al respecto, nos identificamos con Sousa Santos (2007) en cuanto a la generación de conocimiento como "ecología de saberes", revalorizando los saberes puestos en acción de todos los actores involucrados. Para ello resulta fundamental la inclusión activa del conocimiento tradicional-local $\left.\right|^{5} \mathrm{y}$ los procesos de enseñanza acordes con los principios constructivistas (Freire, 1970), de modo de garantizar colectividad, aprendizaje horizontal, discusiones, en una cocreación de conocimiento.

Dado que la agroecología trabaja sobre principios y conceptos en vez de utilizar "recetas", su desarrollo requiere trabajar sobre la singularidad de los sistemas productivos y no es posible hacer generalizaciones de las prácticas. De esta forma, el trabajo debe ser en terreno y desde una perspectiva sistémica, considerando y articulando conocimientos de productores, investigadores y extensionistas. Es así que el fortalecimiento de la agricultura familiar y el diseño de agroecosistemas sustentables deben ser considerados desde una perspectiva socioambiental con un enfoque sistémico y una mirada holística, tal como lo exige la complejidad del proceso (Caporal, 2009).

Una de las preguntas recurrentes que surgieron en los talleres fue cuáles son las estrategias más adecuadas para iniciar los procesos de conversión hacia sistemas de base agro-

5) En los talleres, visitas a campo e intercambio con los productores detectamos varios tipos de saberes de los productores, como el manejo del pastoreo y secuencias de rotaciones, la adaptación de maquinarias para las tareas de siembra, diversificación de cultivos y fechas de siembra para disminuir riesgos, variedades de semillas tradicionales que se guardan y mejoran anualmente, entre tantas otras prácticas que se involucra el conocimiento tradicional-local. 
ecológica. Si bien existe consenso en la necesidad de partir de un diagnóstico del sistema productivo, aun no hay una forma predeterminada de realizarlo, especialmente en cuanto a los aspectos a relevar. La complejidad de los sistemas productivos y la heterogeneidad en los criterios de extensionistas, investigadores y productores, dificulta su realización. El aporte del proyecto a esta temática fue la construcción de una guía de observación y análisis de las unidades de producción desde una perspectiva sistémica con el objeto de detectar puntos débiles sobre los que se debe intervenir para avanzar en el rediseño de los sistemas productivos con base agroecológica. El avance es aún incipiente y se deben hacer varias pruebas y un ajuste a campo para consensuar una metodología de abordaje junto a los productores.

En el marco de este proyecto, las problemáticas planteadas en los sistemas productivos que se acompañaron se fueron contraponiendo con propuestas de solución realizadas en forma conjunta, con una posterior evaluación de resultados. Esto requiere un proceso largo en el tiempo, con avances y retrocesos en función de las posibilidades reales. Por lo tanto, para obtener resultados publicables se precisa un tiempo prolongado, lo que es incompatible con la presión existente dentro del sistema científico para obtener productos en corto plazo y con alto impacto. Las instituciones que pretendan, entonces, consolidar su relación con los agricultores deben tener esto en cuenta y adaptar sus sistemas de evaluación y promoción de investigadores, extensionistas y docentes a estas realidades.

\section{Los faros agroecológicos: una estrategia necesaria}

Otra demanda emergente fue la necesidad de sistematizar las unidades productivas existentes a partir de identificar, ordenar y sociabilizar el conocimiento acumulado en los procesos de conversión hacia sistemas de base agroecológica. Gran parte de las experiencias agroecológicas en el país no está disponible en forma escrita y se conoce solo a través de los relatos orales, interpersonales. Por otro lado, son pocos los casos que dan cuenta de los procesos que han ocurrido a lo largo de la conversión hacia sistemas de base agroecológica y no se presentan las dificultades o riesgos que pueden encontrarse en la implementación de este modelo de producción. Esta falta de sistematización es una fuerte limitación, no solamente para la reflexión y el intercambio de experiencias, sino también para la difusión de casos reales que sirven como demostrativos. Una enseñanza de este proyecto es comprender la necesidad de identificar y sistematizar las experiencias exitosas en la región para conocer sus trayectorias. ${ }^{6}$ Estas producciones fueron fortalecidas a partir del intercambio de saberes ocurrido entre los distintos actores en diferentes espacios. Dichos predios hoy se constituyen en "faros agroecológicos", espacios demostrativos que sirven para promover experiencias de manejo agroecológico, metodología que es necesario profundizar y acompañar su fomento y fortalecimiento (Sánchez Vallduví et al., 2018). Estos casos han sido claves en el cambio de actitud: del "no se puede" a "cómo lo hago". La ubicación y consolidación de estos "faros agroecológicos" es una necesidad a los fines de avanzar en el rediseño de los sistemas productivos hacia un modelo de producción agroecológica. En tanto, la importancia de las interacciones entre productores para la incorporación de tecnologías en las unidades de producción (Monge Perez y Hartwich, 2008) e incluso técnicas formativas entre 
productores que han sido efectivas, como la práctica de campesino a campesino (Holt Giménez, 2008) dan cuenta de la necesidad de contar con casos reales, que muestren a otros productores la posibilidad y ventajas de generar un sistema productivo alternativo al convencional y dominante. Estas iniciativas locales exitosas podrían aportar a la reconfiguración de territorios enteros bajo manejo agroecológico (Altieri y Nichols, 2019).

A mediados de 2019 se habían identificado y acompañado en el marco del proyecto tres experiencias en localidades cercanas a la UNLP (Berisso, San Vicente y Cañuelas), las cuales fueron sistematizadas con el objeto de constituirse como "faros agroecológicos". ${ }^{7}$ Una de las falencias detectadas en este proceso fue la toma de registros, lo cual requirió mucho esfuerzo para que se concretara. Esto ocurrió a pesar del reconocimiento de la necesidad de contar con datos de las producciones locales y sugiere un área de conocimiento vacante sobre la que se debe trabajar. ¿Cuáles son los registros para tomar? ¿Quién/es lo deberían tomar? La toma de registros no es una práctica habitual en los productores familiares, por lo que se debe trabajar con datos sencillos y que sean apropiables. ${ }^{8}$

Por lo tanto, otra de las enseñanzas de la experiencia refiere a que es preciso incorporar actores externos (estudiante-investigador-extensionista) que aborden específicamente esta tarea en articulación con el agricultor. ${ }^{9}$ En este sentido, coincidimos con Bácerez y Díaz (2020), quienes plantean que la participación de los estudiantes y el resto de equipo extensionista en la toma de registros colabora en el proceso formativo, da significado a lo vivenciado y fortalece la producción de conocimiento. ${ }^{10}$

En el marco del proyecto se trabajó en una metodología apropiada para la sistematización de predios agroecológicos que fue desarrollada sobre la base de la experiencia recabada en la interacción con productores y extensionistas en los talleres. La misma fue compartida en el marco de talleres con investigadores, extensionistas y productores, llevados a cabo en el ámbito del 1er. Congreso Argentino de Agroecología (Mendoza, septiembre de 2019) y posteriormente con un grupo de productores en proceso de conversión hacia la agroecología en la localidad de Arrecifes (provincia de Buenos Aires, diciembre de 2019).

\section{La formación de profesionales con una mirada agroecológica}

Una conclusión interesante de los talleres y jornadas fue detectar "la ausencia de técnicos con un enfoque adecuado para elaborar propuestas de conversión y acompañar a los productores en ese proceso". Si bien los estudiantes de la carrera de Agronomía cuentan con una materia obligatoria de Agroecología, ellos buscan desarrollar sus primeras herramientas en el desarrollo profesional a partir del conocimiento y la práctica en casos reales. Lo mismo ocurre con aquellos profesionales que ya se encuentran en el medio laboral y quieren formarse en agroecología. Para acompañar en la formación y apoyar a dicha demanda se

7) Si bien en el marco del proyecto se trabajó con estas tres "faros", existen muchas más experiencias agroecológicas en el territorio.

8) De los talleres realizados surge una crítica en cuanto a la necesidad de una excesiva preocupación por generar datos por los investigadores sin saber para qué, cómo se usa esa información y preguntarse qué es lo que falta saber. 9) De esta forma proponemos trabajar en un marco de Investigación-Acción Participativa (Fals Borda, 2013) que potencie el desarrollo de los faros agroecológicos.

10) Una buena sistematización de un sistema productivo de reconversión fue realizada en un trabajo final de grado por un estudiante de Ciencias Agrarias (Di Piero et al., 2018). 
generaron visitas a sistemas productivos en proceso de conversión hacia sistemas de base agroecológica y giras técnicas a otras regiones productivas. Por otra parte, se efectuaron capacitaciones en temáticas variadas (microbiología de suelo, manejo de pastoreo, producción lechera, etc.) que estuvieron a cargo de miembros del proyecto con experticia en diferentes áreas de las ciencias agrarias y ciencias veterinarias desde un enfoque agroecológico. Estas actividades constituyeron un aporte a la formación de profesionales, aspecto central para el objetivo de desarrollar y amplificar un modelo alternativo de producción agropecuaria con perspectiva agroecológica. A través de estos resultados se percibe claramente que las actividades de extensión contribuyen a la actividad formativa y aportan integralidad a la práctica (Tomassino y Rodríguez, 2011).

Otro de los resultados del proyecto fue comprobar, en los y las estudiantes que participaron, un gran interés por la agroecología y por complementar su formación académica a partir de prácticas a campo. Algunos lo hacen a través de su trabajo final de grado, otros buscan generar el desarrollo de experiencias de puesta en práctica de la agroecología en campos de familiares o bien posicionarse como futuros asesores y asesoras. Se destaca el efecto multiplicador que tuvo la participación en el proyecto para muchos estudiantes, dado que parte de los y las jóvenes integrantes del proyecto se encuentra desarrollando actividades productivas y/o de acompañamiento técnico en sistemas de producción con enfoque agroecológico.

\section{Conclusiones}

El proceso de intervención permitió generar avances en cuanto al desarrollo de la agroecología en los productores familiares y aprendizajes en el equipo técnico para su abordaje en el territorio.

Destacamos la difusión de la propuesta y la incorporación de productores a partir de esta, así como la consolidación de un equipo de trabajo para abordar la conversión a la agroecología en diferentes tipos de producciones. Otro aspecto relevante fue la formación de futuros profesionales que trabajen estas temáticas, con incorporación de estudiantes a las actividades de investigación-extensión y con una perspectiva de futuros multiplicadores de la agroecología.

Un aspecto central fue el intercambio entre productores, docentes (investigadores-extensionistas) y estudiantes a lo largo del proceso que generó una sinergia para un desarrollo, incipiente aún, de la agroecología en el territorio donde se llevó adelante el proyecto. La incorporación de las y los agricultores y organizaciones resultó primordial para abordar procesos de cambios hacia sistemas de base agroecológica. La identificación y acompañamiento de las unidades productivas que se encuentran en un camino de conversión a la agroecología y la generación de información sobre el proceso son claves para el escalamiento de la propuesta. En este sentido, la investigación desde un enfoque participativo y la extensión cumplen una función fundamental.

La experiencia presentada permitió confirmar algunos supuestos o presunciones y entender los cambios que se deben realizar en las instituciones educativas para el desarrollo de la agroecología en los territorios. El desarrollo y difusión de modelos agroecológicos adecuados a las condiciones socioculturales y ambientales de los productores familiares no es sencillo 
bajo el arquetipo de generación-transferencia de tecnología o investigación-extensión-difusión que prevalece en las universidades. Se requiere el desarrollo de nuevos temas de investigación, íntimamente asociados con la extensión y la participación de las y los agricultores. Realizar investigaciones asociadas con los problemas reales de esos agricultores y agricultoras del territorio es todo un aprendizaje, tanto para ellos como para los investigadores e investigadoras. Del mismo modo, es necesario el trabajo conjunto con extensionistas como parte de un proceso transformador basado en metodologías participativas, lo que implica investigación, aprendizaje y acción a partir de la realidad concreta.

Por otro lado, es preciso reflexionar desde un nuevo paradigma de las ciencias agropecuarias en cuanto a las tareas de docencia, investigación y extensión, de manera de buscar la integralidad de la práctica universitaria. Ello demanda un gran esfuerzo en la investigación y la docencia en plena articulación con la práctica de extensión, incorporando diferentes enfoques y metodologías a partir de nuevos interrogantes que surjan de los problemas de las y los agricultores. Resulta fundamental, en este sentido, fomentar, sistematizar y generar conocimiento a partir del intercambio de saberes con productores familiares en territorio, con enfoque agroecológico, y reflexionar sobre cómo esta articulación aporta y retroalimenta a la práctica docente y a la formación de nuevos profesionales de las ciencias agrarias.

\section{Referencias bibliográficas}

Altieri, M; Nicholls, C. (2019). Caminos para la amplificación de la Agroecología. CELIA. https://www.researchgate.net/publication/330967703_Caminos_para_la_amplificacion_de_la_Agroecologia

Bácerez, M. del V. y Díaz, N. (2020). Narrar la experiencia: procesos de registro en las prácticas de extensión universitaria. +E: Revista de Extensión Universitaria, 10(13). e0013. doi: 10.14409/extension.2020.13.Jul-Dic.e0013 Cano, A.; Santos, C.; Tommasino, H. y Stevenazzi F. (2010). De la extensión a las prácticas integrales. https:// www.researchgate.net/publication/263125629_De_la_extension_a_las_practicas_integrales.

Caporal, F. (2009). Extensao Rural e Agroecoloia: temas sobre um novo desenvolvimento rural, necessário e possível. http://www.cpatsa.embrapa.br:8080/public_eletronica/downloads/OPB2444.pdf

Caporal, F. y Costabeber, J. (2004). Agroecologia e extensâo rural. Contribuiçoes para a promoçâo do desenvolvimiento rural sustentável. MDA/SAF/DATER-IICA.

Di Piero, L., Cieza, R. y Cerda, E. (2018). Transición Agroecológica de un Establecimiento de Producción Lechera en la Cuenca de Abasto Sur, Argentina. En Actas Congreso Latinoamericano de Agroecología. Guayaquil. Fals Borda, O.; Herrera Farfán, N.; López Guzmán, L. (Comps.) (2013). Ciencia, compromiso y cambio social. El Colectivo.

Freire, P. (1970). Pedagogía del oprimido. Capítulo I. Siglo XXI Editores.

Holt Giménez, E. (2008). Campesino a campesino: Voces de Latinoamérica Movimiento Campesino para la Agricultura Sustentable. SIMAS. http://www.simas.org.ni/media/1335459671_CaC_Voces_latinoamerica.pdf Monge Pérez, M. y Hartwich, F. (2008). Análisis de Redes Sociales aplicado al estudio de los procesos de innovación agrícola. REDES - Revista hispana para el análisis de redes sociales, 14(1), 1-31.

Patrouilleau, M.; Martínez, C.; Cittadini, E.; Cittadini, R. (2017). Políticas públicas y desarrollo de la agroecología en Argentina. En Sabourin, E. (Org.). Políticas públicas a favor de la agroecología en América Latina y El Caribe. Evangraf / Criação Humana, Red PP-AL. FAO.

Sousa Santos, B. de (2007). La universidad del Siglo XXI. Para una reforma democrática y emancipadora de la universidad. CIDES-UMSA, ASDI y Plural editores. 
Sánchez Vallduví, G., Principi, G., Dure S., Eirin, M., y Giordano, G. (2018). Producción agroecológica de un tambo familiar en Uribelarrea. Fortalecimiento del proceso de transición y construcción de un espacio demostrativo. VI Jornada de Extensión del Mercosur. 25-27 de abril. Tandil, Argentina. http://extension.unicen.edu. ar/jem/subir/uploads/2018_313.pdf

Sarandón, S.J. y C.C. Flores. (2014). La insustentabilidad del modelo agrícola actual. En: Agroecología: Bases teóricas para el diseño y manejo de agroecosistemas sustentables. Sarandón, S.J. y C.C. Flores (comp) Edulp. Buenos Aires, Argentina. pp: 13-41 http://sedici.unlp.edu.ar/handle/10915/37280

Sociedad Argentina de Agroecología (2020). ¿Qué nos propone la agroecología? Documento interno.

Tomassino. H; Rodriguez, N. (2011). Tres tesis básicas sobre extensión y prácticas integrales de la Universidad de la Republica. En Cuadernos de Extensión. Universidad de la Republica. Uruguay. 